\title{
Perfil do conhecimento e comportamento sexual de adolescentes
}

\author{
Knowledge profile and sexual behaviors of adolescents
}

Perfil de conocimiento y comportamientos sexuales de adolescentes

\begin{abstract}
Inara Rege Lopes ${ }^{1}$, Alisséia Guimarães Lemes ${ }^{1,2}$, Marcos Vinicius Costa Santos ${ }^{1}$, Andre Cantarelli Vilela $^{1}$, Suzicléia Elizabete de Jesus Franco ${ }^{1}$, Aline Aparecida Rodrigues ${ }^{1}$, Thiago Lopes Brito ${ }^{1}$, Elias Marcelino da Rocha ${ }^{1 *}$.
\end{abstract}

\section{RESUMO}

Objetivo: Identificar o conhecimento e o comportamento sexual de adolescentes no interior de Mato Grosso. Métodos: Estudo transversal, exploratório descritivo e quantitativo, realizado com 57 adolescentes do sexo masculino no ano de 2018, estudantes em uma escola de ensino médio localizada em um município na região Centro-Oeste, no estado de Mato Grosso que foram avaliados por meio de um questionário semiestruturado. Resultados: A análise dos dados revelou que os adolescentes possuem conhecimento sobre sexualidade, embora eles reportaram não ter a liberdade necessária para falarem sobre a temática no âmbito familiar, recorrendo a fontes duvidosas, como a internet e amigos. Por outro lado, os resultados apontaram para uma pratica sexual insegura entre eles, com destaque para o não uso de preservativo nas relações sexuais e a pratica de sexo sob efeito de álcool. Conclusão: Os resultados demonstram a necessidade da realização de atividades que envolvam a promoção da saúde e a prevenção de doenças que atenda as dúvidas sobre sexualidade dos adolescentes, estendendo aos familiares e professores. Assim, acredita-se que esse grupo da população terá a compreensão necessária para desenvolver um comportamento sexual seguro, ampliando assim o seu autocuidado de sua saúde sexual.

Palavras-chave: Adolescentes, Comportamento sexual, Estudantes, Sexualidade.

\begin{abstract}
Objective: To identify the knowledge and sexual behavior of adolescents in the interior of Mato Grosso. Methods: Cross-sectional, exploratory, descriptive and quantitative study, carried out with 57 male adolescents in 2018, students at a high school located in a municipality in the Midwest region, in the state of Mato Grosso, which were evaluated using a semi-structured questionnaire. Results: The analysis of the data revealed that the adolescents have knowledge about sexuality, although they reported not having the necessary freedom to talk about the topic within the family, using dubious sources, such as the internet and friends. On the other hand, the results pointed to an unsafe sexual practice among them, with emphasis on the non-use of condoms during sexual intercourse and the practice of sex under the influence of alcohol. Conclusion: The results demonstrate the need to carry out activities that involve health promotion and disease prevention that meets the doubts about sexuality among adolescents, extending to family members and teachers. Thus, it is believed that this group of the population will have the necessary understanding to develop safe sexual behavior, thus expanding their self-care for their sexual health.
\end{abstract}

Keywords: Adolescents, Sexual behavior, Students, Sexuality.

\section{RESUMEN}

Objetivo: Identificar el conocimiento y el comportamiento sexual de los adolescentes en el interior de Mato Grosso. Métodos: Estudio transversal, exploratorio, descriptivo y cuantitativo, realizado con 57 adolescentes varones en 2018, estudiantes de una escuela secundaria ubicada en un municipio de la región del Medio Oeste, en el estado de Mato Grosso, que fueron evaluados utilizando Un cuestionario semiestructurado. Resultados: El análisis de los datos reveló que los adolescentes tienen conocimiento sobre la sexualidad, aunque informaron que no tenían la libertad necesaria para hablar sobre el tema dentro de la familia, utilizando

\footnotetext{
1 Universidade Federal de Mato Grosso (UFMT), Mato Grosso-Brasil. *E-mail: eliasufmt@yahoo.com.br.

${ }^{2}$ Escola de enfermagem de Ribeirão Preto da Universidade de São Paulo (EERP-USP), Ribeirão Preto-SP.
} 
fuentes dudosas, como Internet y amigos. Por otro lado, los resultados apuntaron a una práctica sexual insegura entre ellos, con énfasis en el no uso de condones durante las relaciones sexuales y la práctica del sexo bajo la influencia del alcohol. Conclusión: Los resultados demuestran la necesidad de llevar a cabo actividades que involucren la promoción de la salud y la prevención de enfermedades que satisfagan las dudas sobre la sexualidad entre los adolescentes, extendiéndose a los familiares y maestros. Por lo tanto, se cree que este grupo de la población tendrá la comprensión necesaria para desarrollar un comportamiento sexual seguro, expandiendo así su autocuidado para su salud sexual.

Palabras clave: Adolescentes, Comportamiento sexual, Estudiantes, Sexualidad.

\section{INTRODUÇÃO}

De acordo com o Estatuto da Criança e do Adolescente, adolescente é o indivíduo de 12 a 18 anos de idade, já o Ministério da Saúde (MS) segue a definição prescrita pela Organização Mundial da Saúde (OMS), considera adolescentes aqueles que têm entre 10 e 19 anos (ECA, 2019; BRASIL, 2018).

A adolescência é uma fase de transferência que ocorre entre a infância e a vida adulta, e esta é marcada por modificações corporais, mentais, sociais, emocionais e sexuais, onde o indivíduo concretiza seu desenvolvimento, tornando-se independente, e assumindo o seu papel na sociedade (EISENSTEIN E, 2015). Há certa confusão acerca do que é puberdade e o que é adolescência, pois ambos ocorrem praticamente ao mesmo tempo, porém a puberdade é o conjunto de processos biológicos que resultam no amadurecimento dos órgãos sexuais, enquanto a adolescência, compreende além disto, as mudanças psicológicas e sociais que ocorrem nesta fase (CAMPAGNA VN e SOUZA ASL, 2006).

Em meio as intensas modificações corporais, a sexualidade é um aspecto central na vida do ser humano, e envolve vários fatores como, a orientação sexual, o erotismo, o prazer, a afetividade, a prática sexual e a reprodução. Ela se constrói desde a infância, influenciada no ambiente onde se vive, podendo ter seu significado modificado conforme a religião, crenças, costumes, normas éticas e morais em que o indivíduo está inserido (BRASIL, 2018).

Vale ressaltar que a sexualidade não se resume ao "ato sexual", mas envolve um processo de busca e troca, estando presente na vida do ser humano desde o seu nascimento e se aperfeiçoando em cada fase da vida, e é considerado um fator importante para se obter qualidade de vida (NERY IS, et al. 2015).

Em busca de ofertar uma melhor compreensão sobre a sexualidade aos adolescentes torna-se necessário que estes recebam orientações que possam contribuir para uma pratica sexual segura e essas informações prioritariamente devem acontecer em locais considerados de confiança, como o ambiente familiar, os serviços de saúde e na escola.

Com vistas a planejar ações efetivas que atinjam essa população é necessário elaborar estratégias que visem obter o conhecimento prévio deles sobre o assunto. Assim, este estudo teve como objetivo identificar o conhecimento e o comportamento sexual de adolescentes no interior de Mato Grosso.

\section{MÉTODOS}

Trata-se de um estudo transversal, exploratório descritivo, com abordagem quantitativa, realizado com adolescentes do sexo masculino que frequentavam uma escola em um município localizado na região CentroOeste, no estado de Mato Grosso, que atenderam os critérios estabelecidos para este estudo.

Os sujeitos do estudo foram compostos por estudantes regularmente matriculados no ensino médio de uma escola estadual localizada no interior de Mato Grosso. Esta escola foi selecionada por participar de uma ação de extensão sobre "Saúde Sexual", realizada por membros do projeto de extensão "Pró-Homem" vinculado a Universidade Federal de Mato Grosso (UFMT), Campus Universitário do Araguaia (CUA).

Existiam no momento da coleta de dados 10 salas do ensino médio com aproximadamente 313 alunos, destes 105 eram do sexo masculino. Deste total, atenderam os critérios estabelecidos para o estudo 57 adolescentes que foram selecionados por conveniência. Participaram do estudo e da ação apenas os alunos do $1^{\circ}$ e $2^{\circ}$ ano do ensino médio. 
Os critérios de inclusão foram: estudantes do sexo masculino, com idade de 15 a 18 anos, devidamente matriculados na escola participante do estudo. Sendo excluídos os estudantes que não estiveram presentes na sala de aula no momento da pesquisa e os questionários preenchidos de forma incompleta.

Os dados foram coletados no período de outubro a novembro de 2018, por meio da aplicação de um questionário semiestruturado, contendo questões objetivas que abordaram aspectos sobre a identificação dos participantes e do conhecimento e comportamento sexual dos estudantes.

Os adolescentes responderam o questionário nas salas de aula, após a permissão da direção e professores e após preenchimento do Termo de Consentimento Livre e Esclarecido que foi assinado pelo responsável legal e pelo estudante. A aplicação do questionário teve a duração média de 20 minutos.

Os dados foram lançados e organizados no programa Epi Info versão 3.5.2. A análise ocorreu de forma descritiva e os resultados foram apresentados em tabelas.

A pesquisa foi aprovada pelo Comitê de Ética em Pesquisa com Seres Humanos da Universidade Federal de Mato Grosso, sob número CAAE: 65604317.2.0000.5587 e protocolo no 2.062.048, respeitando a Resolução nำ466/12 do Conselho Nacional de Saúde (CNS).

\section{RESULTADOS}

Participaram do estudo 57 adolescentes do sexo masculino, com idade entre 15 a 18 anos e média de 16,08 anos, predominando estudantes do primeiro ano do ensino médio (68\%), conforme apresentado na Tabela 1.

Destes dados vão emergir os parâmetros que normalmente são advindos da puberdade, como os caracteres secundários sexuais vivenciados na puberdade e nesta fase escolar que irão acontecer as interações sociais levando os adolescentes as experiências da juventude e a maturidade do mundo adulto.

Tabela 1 - Distribuição da idade e da série do ensino médio cursada pelos entrevistados, $(n=57)$. Região Centro-Oeste, do estado de Mato Grosso, Brasil, 2018.

\begin{tabular}{lll}
\hline Descrição & $\mathbf{N}$ & $\%$ \\
\hline Idade & & \\
\hline 15 anos & 14 & 24,6 \\
16 anos & 26 & 45,6 \\
17 anos & 15 & 26,3 \\
18 anos & 2 & 3,5 \\
\hline Total & 57 & $100 \%$ \\
\hline Série do ensino médio & & $68 \%$ \\
\hline $1^{\circ}$ ano do ensino médio & 39 & $32 \%$ \\
20 ano do ensino médio & 18 & $100 \%$ \\
\hline Total & 57 & \\
\hline
\end{tabular}

Fonte: Lopes IR, et al., 2020.

\section{Conhecimento dos estudantes adolescentes sobre sexualidade}

Neste estudo observou que os adolescentes avaliam seu nível de conhecimento sobre a sexualidade como médio (58\%) e alto (24\%), conforme apresentado no Gráfico 1.

Evidencia que na fase da adolescência há dificuldade de compreender os sentimentos emocionais, devido transformações hormonais e a transição da fase infantil para adolescência, passando por modificações que levarão ao autoconhecimento do próprio corpo. 
Gráfico 1 - Distribuição do nível de conhecimento sobre sexualidade na percepção dos estudantes adolescentes, ( $n=57)$. Região Centro-Oeste, do estado de Mato Grosso, Brasil, 2018.

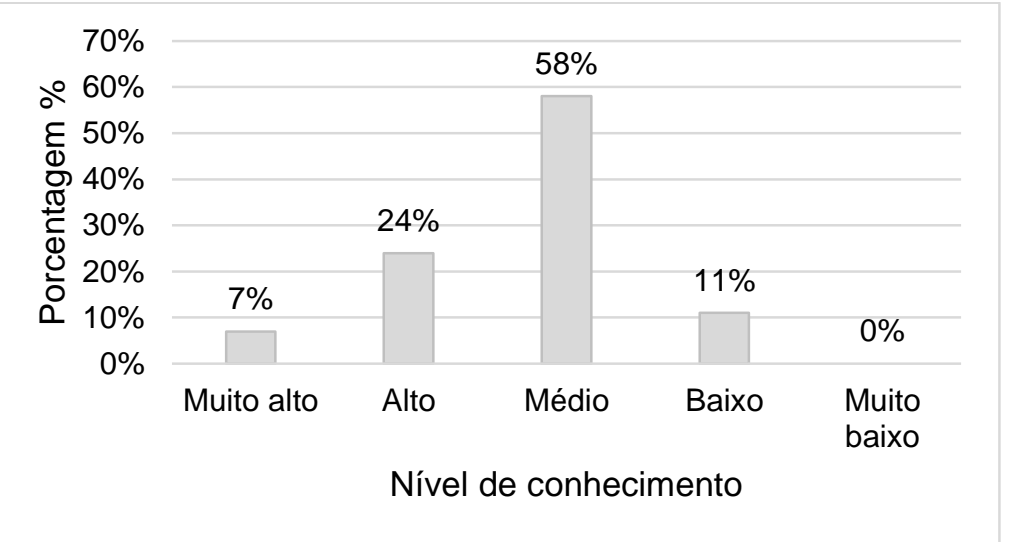

Fonte: Lopes IR, et al., 2020.

Na Tabela 2 verifica-se que o nível de abertura para conversar sobre sexualidade com os familiares responsáveis que residem com os estudantes variou de nem aberto e nem fechado para o diálogo (49\%) e aberto (32\%). Enquanto que o nível de qualidade dessas conversas no ambiente familiar foi considerado pelos estudantes como nem bom ou mal (51\%) e bom (41\%). Observou-se que os estudantes recorrem como fonte de informação quando querem saber sobre temas relacionados a sexualidade predominantemente a internet (82\%), seguido de amigos (42\%), mãe (32\%) e pai $(30 \%)$ e a palestras $(25 \%)$ (Tabela 2 ).

Tabela 2 - Distribuição do nível de abertura e qualidade para diálogo no ambiente familiar e fonte de busca por informações sobre sexualidade, ( $n=57)$. Região Centro-Oeste, do estado de Mato Grosso, Brasil, 2018.

\begin{tabular}{lll}
\hline Descrição & N & $\%$ \\
\hline Nível de abertura para o diálogo sobre sexualidade no ambiente familiar & \\
\hline Muito fechado & 2 & $3 \%$ \\
Fechado & 3 & $5 \%$ \\
Nem fechado nem aberto & 28 & $49 \%$ \\
Aberto & 18 & $32 \%$ \\
Muito aberto & 6 & $11 \%$ \\
\hline Total & 57 & $100 \%$ \\
\hline Nível de qualidade do diálogo sobre sexualidade & no ambiente familiar & \\
\hline Muito mau & 2 & $03 \%$ \\
Mau & 2 & $03 \%$ \\
Nem mau nem bom & 29 & $51 \%$ \\
Bom & 23 & $41 \%$ \\
Muito bom & 1 & $02 \%$ \\
\hline Total & 57 & $100 \%$ \\
\hline Fonte de busca por informações sobre sexualidade & \\
\hline Internet & 47 & $82 \%$ \\
Amigos & 24 & $42 \%$ \\
Mãe & 18 & $32 \%$ \\
Pai & 17 & $30 \%$ \\
Palestras & 14 & $25 \%$ \\
TV & 11 & $19 \%$ \\
Professores & 9 & $16 \%$ \\
Filmes & 8 & $14 \%$ \\
Irmãos & 4 & $0,7 \%$ \\
Jornal & 4 & $0,7 \%$ \\
Revistas & 3 & $0,5 \%$ \\
\hline Fonte: & &
\end{tabular}

Fonte: Lopes IR, et al., 2020. 


\section{Comportamento sexual dos estudantes adolescentes}

No Gráfico 2, verificou-se o predomínio de adolescentes que já tiveram sua primeira relação sexual (58\%), sendo a idade desse primeiro contato entre 10 a 17 anos, com destaque para 14 anos (14\%) e 15 anos (16\%), conforme demonstrado na Tabela 3.

Gráfico 2 - Distribuição das respostas dos adolescentes quanto já ter tido relação sexual, $(n=57)$. Região Centro-Oeste, do estado de Mato Grosso, Brasil, 2018.

$70 \%$

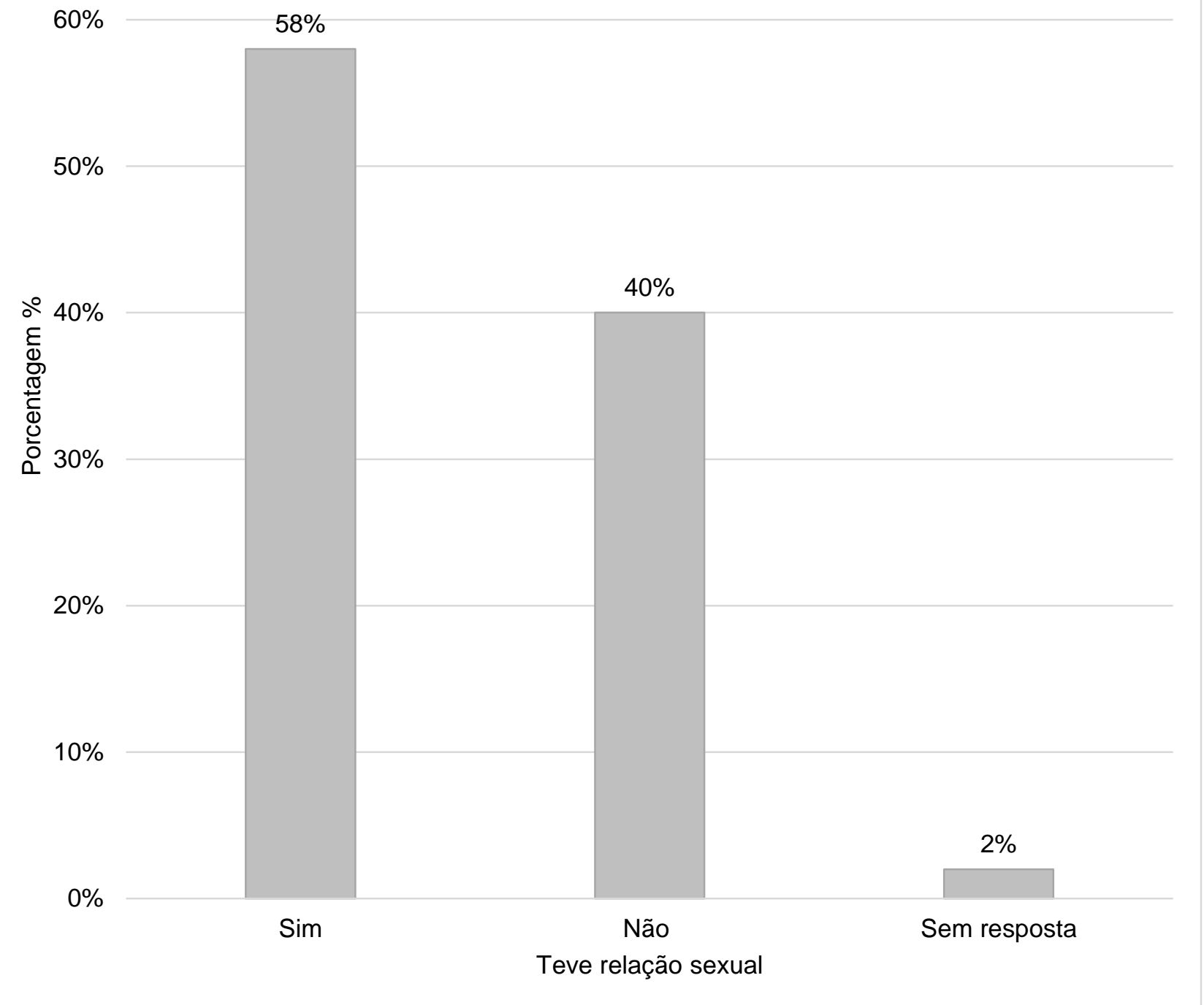

Fonte: Lopes IR, et al., 2020.

A Tabela 3 também destaca que parte dos estudantes (10\%) assumiram sentir pressionados para ter relação sexual.

Além disso, observou-se que uma parte significativa dos alunos (33 adolescentes) já tiveram sua primeira experiência com o sexo e o que chama a atenção é que 39\% não fizeram uso de nenhum tipo de método contraceptivo, incluindo o uso da camisinha, embora $82 \%$ dos meninos afirmaram ter receio de uma gravidez indesejada e não planejada pela parceria. 
Tabela 3 - Distribuição do comportamento sexual dos estudantes adolescentes, ( $n=57)$. Região Centro-Oeste, do estado de Mato Grosso, Brasil, 2018.

\begin{tabular}{|c|c|c|}
\hline Descrição & $\mathbf{N}$ & $\%$ \\
\hline \multicolumn{3}{|c|}{ Idade da primeira relação sexual } \\
\hline 10 anos & 2 & $3 \%$ \\
\hline 12 anos & 3 & $5 \%$ \\
\hline 13 anos & 5 & $9 \%$ \\
\hline 14 anos & 8 & $14 \%$ \\
\hline 15 anos & 9 & $16 \%$ \\
\hline 16 anos & 2 & $3 \%$ \\
\hline 17 anos & 1 & $2 \%$ \\
\hline Sem resposta & 27 & $47 \%$ \\
\hline Total & 57 & $100 \%$ \\
\hline \multicolumn{3}{|c|}{ Uso método contraceptivo na primeira relação } \\
\hline Sim & 12 & $21 \%$ \\
\hline Não & 22 & $39 \%$ \\
\hline Sem resposta & 23 & $40 \%$ \\
\hline Total & 57 & $100 \%$ \\
\hline \multicolumn{3}{|c|}{ Receio de uma gravidez não planejada } \\
\hline Sim & 47 & $82 \%$ \\
\hline Não & 9 & $16 \%$ \\
\hline Sem resposta & 1 & $2 \%$ \\
\hline Total & 57 & $100 \%$ \\
\hline \multicolumn{3}{|c|}{$\begin{array}{l}\text { Relação sexual ocasional sem uso do } \\
\text { preservativo }\end{array}$} \\
\hline Sim & 25 & $44 \%$ \\
\hline Não & 11 & $19 \%$ \\
\hline Sem resposta & 20 & $35 \%$ \\
\hline Total & 57 & $100 \%$ \\
\hline \multicolumn{3}{|c|}{ Já fez sexo sob efeito do álcool } \\
\hline Sim & 9 & $16 \%$ \\
\hline Não & 24 & $42 \%$ \\
\hline Sem resposta & 24 & $42 \%$ \\
\hline Total & 57 & $100 \%$ \\
\hline
\end{tabular}

Fonte: Lopes IR, et al., 2020.

Observou ainda neste estudo que os adolescentes apresentaram comportamento sexual de risco, como a afirmação que $44 \%$ destes já tiveram uma relação sexual ocasional sem se importar em utilizar o preservativo e também pelo fato de $16 \%$ dos estudantes já ter feito sexo sob efeito de bebida alcoólica, como também demostrado na tabela 3.

\section{DISCUSSÃO}

As características sociodemográficas verificados nesta pesquisa foram semelhantes a outros estudos nacionais e internacionais (ORCASITA LT, et al., 2018; SOUSA BC, et al., 2018; GENZ N, et al., 2017; VASCONCELOS ACS, et al., 2016).

Os autores acima citados, mencionam ainda que as transformações corporais acontecem principalmente dos 12 aos 15 anos de idade e na mesma fase escolar. Momento que muitos dos adolescentes também tem suas primeiras experiencias do autoconhecimento corporal e vivenciam sentimentos de desejos sexuais que até o momento não tinham sido despertados. 
A adolescência e suas alterações e vulnerabilidades pode acarretar uma fase delicada na vida do indivíduo. Seus atos vão gerar consequências muitas vezes irreparáveis para sua vida futura e também para toda a sociedade (MORAES SP, VITALLE MSS, 2015). Desta forma, identificar como os adolescentes compreendem e vivenciam sua saúde sexual, poderá facilitar na elaboração de medidas orientativas que contribuirá para reduzir os riscos que estes estão expostos mediante a uma vida sexual insegura.

Neste estudo a maioria dos adolescentes referiram ter um conhecimento satisfatório (muito alto, alto e médio) sobre sua sexualidade, assim como encontrado em um estudo realizado com adolescente no município de Embu (SP), onde 49\% dos meninos consideram suficiente seu grau de conhecimento sobre sexo e sexualidade (BRETAS JRS, et al., 2011).

Embora ambos os estudos apontam para um conhecimento satisfatório dos estudantes quanto a sexualidade, é importante destacar que parte destes podem apresentar dificuldade e resistência em falar sobre o próprio corpo, podendo estar relacionado às concepções sobre a masculinidade e às relações dessas com o cuidado em saúde (VASCONCELOS ACS, et al., 2016).

Em busca de ter um conhecimento adequado sobre a sexualidade é necessário que os adolescentes encontrem espaços que promovam o diálogo, com vistas a receber orientações devidas referente a essa temática. Um dos espaços considerado relevante para que esse diálogo aconteça é o próprio ambiente familiar.

Neste estudo, uma parcela pequena dos estudantes consideraram ter um ambiente familiar aberto e de qualidade para falar sobre sua sexualidade. Dados semelhantes foram encontrado em uma pesquisa realizada na Colômbia com meninos adolescentes, que reportaram uma certa dificuldade para falar sobre sexualidade com seus pais, principalmente entre os estudantes de escola privada (30.4\%), quando comparado com os alunos de escola pública (18.7\%) (ORCASITA LT, et al., 2018).

Ambos os resultados revelam que ainda no século XXI existem barreiras que podem prejudicar um diálogo construtivo entre pais e filhos. Uma dessas barreiras deve-se ao fato de que os pais muitas vezes embasavam na crença de que conversar sobre a sexualidade poderá induzir seu filho (a) a pratica-lo, por esse motivo, procuraram preservar o silêncio sobre esse assunto (GONÇALVES RC, FALEIRO JH, MALAFAIA G, 2013; QUEIRÓS PS, et al., 2016).

A comunicação fechada no ambiente familiar pode estar relacionada ao autoritarismo por parte dos pais, que faz com que os filhos não se sintam à vontade para conversar e tirar dúvidas, ou que os pais se sentem incapazes intelectualmente e emocionalmente para falar sobre o assunto com seus filhos (QUEIRÓS PS et al., 2016).

Em um estudo foi reportado que a figura paterna está mais ausente no acompanhamento do adolescente, tanto no dialogo quanto na busca por atenção em saúde sexual (ORCASITA LT, et al., 2018). Esse tipo de comportamento familiar, pode favorecer que esses meninos passem a preferir falar sobre questões que envolvem a sexualidade com outras pessoas que não seus pais, pelo fato de que falar com os pais, indicaria a necessidade de aprovação para começar sua vida sexual (ORCASITA LT, et al., 2018).

Frente a importância de se estabelecer um espaço de respeito para o diálogo entre filho e pais com vistas a tratar de assuntos que envolva seu crescimento e desenvolvimento, como a sexualidade, uma pesquisa internacional realizada no norte de Portugal revelou que a qualidade da relação que os adolescentes estabelecem com seus pais, torna-se muito importante para que tenha uma comunicação efetiva (BARREIRA IMB, et al., 2015).

Mais que isso, a influência que os pais possuem sobre os filhos, devem-se em grande parte à qualidade da relação que estes estabelecem com eles, o que pode favorecer a resolução de problemas, funcionando como uma fonte de suporte e apoio mais importante até que os amigos BARREIRA IMB, et al., 2015).

Essas barreiras na comunicação entre pais e filhos fazem com que os adolescentes procurem informações em outros meios de comunicação, que são consideradas como fontes de informações imprecisas ou incompletas, que não tem uma real confiabilidade. Além disso, buscando através destes meios pode se encontrar informações que não estão inseridas na realidade destes jovens, levando-os a uma não compreensão ou a um equívoco destas, contribuindo para a prática sexual insegura (NERY IS, et al., 2015; ORCASITA LT, et al., 2018). 
Estudos que abordaram sobre a comunicação entre pais e adolescentes (NERY IS, et al., 2015; QUEIRÓS OS, et al., 2016) revelam a importância do diálogo na formação de opinião do adolescente e no cuidado com sua saúde, que quando não é bem orientado sobre os cuidados que se deve tomar ao iniciar a sua vida sexual, o leva a práticas sexuais de risco que podem trazer consequências irreversíveis para o futuro, mas que em contrapartida, quando há um diálogo saudável entre pais e filhos, os adolescentes se tornam mais reflexivos e críticos na tomada de decisões.

Quanto aos recursos buscados pelos adolescentes como meio de obter informações sobre a temática sexualidade, no presente estudo os estudantes referiram preferencialmente buscar essas informações na internet e entre amigos, posteriormente entre os pais ou em palestras.

Comportamento divergente foi verificado entre adolescente de um estudo realizado no Rio Grande do Sul (BR), onde estes buscavam como medida de informação fontes consideradas seguras, como os pais, os irmãos, os professores e os serviços de saúde (GENZ N, et al., 2017), fato também verificado em um estudo colombiano, onde preferencialmente os adolescentes recorriam como fonte de informação os familiares $(41,9 \%)$, profissionais de saúde $(33,1 \%)$, professores $(28,4 \%)$, seguido da internet $(41,2 \%)$, amigos $(38,1 \%)$ e televisão (37,2\%) (ORCASITA LT, et al., 2018).

Em um estudo foi relatado que na atualidade os jovens têm facilidade de acesso à informação e ao conhecimento, através de diferentes fontes: internet, livros, revistas; porém, continua sendo indispensável o diálogo e a discussão com pessoas capacitadas e disponíveis para esclarecê-los e ajudá-los no entendimento de sua sexualidade (FREITAS KR, DIAS SMZ, 2010).

Quanto a pratica sexual entre os adolescentes entrevistados neste estudo, verificou-se que a maioria destes (58\%) já tiveram sua primeira relação sexual, entre 10 a 17 anos, com destaque para 14 e 15 anos.

Esse mesmo comportamento também foi verificado em um estudo Matogrossense com 499 alunos, onde $51,3 \%$ dos meninos tiveram a primeira pratica sexual com penetração dos 14 aos 16 anos e $39,1 \%$ dos 12 aos 13 anos, 7,3\% dos 8 aos 10 anos e 2,4\% dos 17 aos 20 anos (LIMA FCA, et al., 2013).

Em duas pesquisas realizadas em âmbito nacional revelaram que a iniciação sexual de adolescentes foi semelhante ao verificado neste estudo.

A primeira, considerada como Pesquisa Nacional de Saúde do Escolar (PeNSE) que avaliou 10926 questionários preenchidos por escolares de 13 a 17 anos de idade, identificou que $60 \%$ dos meninos já tiveram seu primeiro contato sexual entre 16 e 17 anos, enquanto que $34,5 \%$ com 13 a 15 anos (IBGE, 2015).

A segunda, uma pesquisa realizada em escolas de cidades brasileiras com 75.060 estudantes de 12 a 17 anos, identificou-se que $44,2 \%$ da amostra iniciaram a vida sexual aos 16 anos e $52,4 \%$ aos 17 anos (BORGES ALV, et al., 2016).

A iniciação sexual precoce implica em aumento da incidência de relações sexuais de risco, violência sexual, uso inadequado de métodos contraceptivos, aumento da incidência de gravidez, e aumento do risco de adquirir Infecções Sexualmente Transmissíveis (IST's) (LARA LAS, ABDO CHN, 2015; MORAES L, 2019).

Foi averiguado em um estudo informações acerca de que um estudante apresenta histórico sexual precoce, torna-se necessário uma maior vigilância dos serviços que trabalham diretamente com crianças e adolescentes, como as escolas e as equipes da Estratégia de Saúde da Família, com vistas a identificar possíveis sinais de abuso sexual e tomarem as devidas providencias, como efetuarem a notificação e informarem as autoridades locais, como Conselhos Tutelares e Ministério Público (SOUSA BC, et al., 2018).

É importante destacar que a iniciação sexual é determinada por vários fatores, dentre eles, nível de escolaridade, condições financeiras e socioculturais, exposição precoce a cenas eróticas, falta de orientação, má estruturação familiar (LARA LAS, ABDO CHN, 2015).

Para que os adolescentes iniciem práticas sexuais de maneira segura e saudável é necessário a união de fatores como: diálogo familiar, educação em saúde da comunidade e orientação sobre práticas seguras nas escolas desde a puberdade até pré-adolescência (BRASIL, 2017).

Assim, a aproximação dos professores e profissionais da saúde é de suma relevância com vistas a facilitar a compreensão das informações recebidas (VALLI GP, COGO ALP, 2013). 
Embora os adolescentes deste estudo afirmaram possuir um nível significativo de conhecimento sobre o assunto sexualidade e até mesmo terem receio de uma gravidez indesejada, verificou-se em suas respostas contradições a essas afirmações, pois, parte desses meninos assumiram não ter usado preservativo na primeira relação sexual (39\%), já ter feito sexo ocasional sem o uso de preservativo (44\%) e até mesmo ter tido relação sexual sob de efeito de bebida alcoólica (16\%), o que reflete em um comportamento de risco que pode acarretar prejuízos no âmbito individual e coletivo.

Paralelamente, um estudo realizado na Bahia com 811 adolescentes revelou que 21,3\% da amostra não utilizaram o preservativo na última relação sexual (SOUSA BC, et al., 2018), assim como foi verificado em um estudo realizado com adolescentes em Cuiabá, onde 24\% também não utilizaram nenhum método contraceptivo, incluindo o preservativo (LIMA FCA, et al., 2013).

Esses resultados se contrapõem ao encontrado na PeNSE de 2015, onde evidenciou que mais da metade dos escolares masculinos afirmaram ter utilizado preservativo na primeira vez em que tiveram relação sexual (56,8\%) (IBGE, 2015).

Quanto ao sexo sob efeito de álcool, um estudo reportou que adolescentes que experimentaram uma dose de álcool tiveram 1,41 vezes mais prevalência de relações sexuais (SOUZA BC, et al., 2018), o que pode contribuir para uma pratica sexual insegura e de riscos, que pode trazer prejuízos irreversíveis, o mesmo ocorre para a pratica sexual sem uso de preservativo.

Desta forma, é crucial conhecer o seu próprio corpo e o da sua parceria, sendo também importante a utilização de métodos contraceptivos, pois contribuem para ato sexual saudável e seguro. O preservativo masculino é o método contraceptivo mais conhecido entre os adolescentes e sua utilização cresceu muito no Brasil nos últimos anos, pois seu uso não se restringe a casais ou pessoas que possuem parceria fixa, sendo também utilizado por pessoas que possuem múltiplas parcerias, pois sua finalidade não se restringe a prevenção de gravidez indesejada, mas também a prevenção de IST's (MOLA R, 2016; OLSEN JM, 2018, CUMBER SN, TSOKA-GWEGUWENIL JM, 2016).

Mediante ao exposto, acredita-se que a pouca experiência dos adolescentes pesquisados sobre a vivencia das práticas sexuais, pode estar relacionado a falta de educação sexual. Autores enfatizaram que os adolescentes não têm conhecimento suficiente acerca do assunto, o que sugere que adolescentes não estão tendo informações de boa qualidade. Para tanto há necessidade de articulação entre a família, escolas, igrejas e serviços de saúde, afim de que tenha grandes investimentos sobre sexualidade, educação sexual, práticas sexuais e métodos de prevenção, destacando vantagens e desvantagens (PORTELA NLC, ARAÚJO LP, 2013).

A escola é citada como uma fonte segura para repassar informações sobre sexualidade aos adolescentes. Esse espaço é visto como o melhor ambiente para se trabalhar ações de educação sexual com esse grupo, tendo em vista que estes passam um tempo considerável neste espaço, acreditando que é para lá que eles levam suas dúvidas e esperam que essas sejam respondidas (CHAVES ACP, et al., 2014).

Desta forma, acredita-se que quando uma pessoa detém de conhecimento sobre sua sexualidade, isso significa que já foi sensibilizada e conscientizada sobre a importância do uso correto de preservativos, cuidados com a saúde, sobre gravidez indesejada e bem-estar psicoemocional. As informações corretas poderão tornar os adolescentes em pessoas mais seguras, menos tímidas, mais responsáveis e capazes de confiar em seus atos, tornando as práticas sexuais mais seguras e prazerosas.

\section{CONCLUSÃO}

O presente estudo permitiu identificar que os adolescentes apesar de referirem conhecer sobre sexualidade, pouco colocavam em prática o que sabiam, pois, a maioria apresenta um comportamento de risco em suas práticas sexuais, como o não uso de preservativos nas relações sexuais. Destaca-se que é de suma importância que esses adolescentes encontrem no ambiente da família espaço para o diálogo e assim sanar suas dúvidas, pois considera-se que falar sobre o assunto é um fator determinante para a melhor interpretação e conduta para uma pratica sexual segura. Para que o tema sexualidade deixe de ser considerado tabu, é necessário que possa ser (re)pensado dentro do ambiente familiar, escolar, e na sociedade em geral, com o objetivo de sensibilizar as pessoas acerca de práticas sexuais saudáveis. 


\section{REFERÊNCIAS}

1. BARREIRA IMB, et al. Cultura organizacional da família como preditor das atitudes e comportamentos sexuais em adolescentes. Revista de Enfermagem Referência, 2015; - IV - n. ${ }^{\circ} 6$-pp. 17-25.

2. BORGES ALV, et al. ERICA: início da vida sexual e contracepção em adolescentes brasileiros. Revista de Saúde Pública, 2016; 50(supl 1):15s.

3. BRASIL. [Estatuto da criança e do adolescente (1990) ]. Estatuto da criança e do adolescente: Lei n. 8.069, de 13 de julho de 1990, e legislação correlata [recurso eletrônico] / Fabio Vaisman (organizador). - 17. ed. - Brasília: Câmara dos Deputados, Edições Câmara, 2019 - (Série legislação; n. 260 e-book).

4. BRASIL. Ministério da Saúde. Secretaria de Atenção à Saúde. Departamento de Ações Programáticas e Estratégicas. Proteger e cuidar da saúde de adolescentes na atenção básica / Ministério da Saúde, Secretaria de Atenção à Saúde, Departamento de Ações Programáticas e Estratégicas. - 2. ed. - Brasília: Ministério da Saúde, 2018.

5. BRETAS JRS, et al. Aspectos da sexualidade na adolescência. Ciência \& Saúde Coletiva, 2011; 16(7):3221-3228.

6. CAMPAGNA VN, SOUZA ASL. Corpo e imagem corporal no início da adolescência feminina. Boletim de Psicologia, 2006; vol.56, n.124, pp. 9-35.

7. CHAVES ACP, et al. Conhecimentos e atitudes de adolescentes de uma escola pública sobre a transmissão sexual do HIV. Revista Brasileira de Enfermagem, 2014; 67(1): 48-53.

8. CUMBER SN, TSOKAGWEGWENI JM. Knowledge and practice of condom use as well as perceived barriers among street adolescents in Cameroon. S Afr J HIV Med. 2016;17(1), a479.

9. EISENSTEIN E. Adolescência: definições, conceitos e critérios. Adolescência e Saúde, 2015; 2(2):6-7.

10. FREITAS KR, DIAS SMZ. Percepções de adolescentes sobre sua sexualidade. Texto e Contexto Enfermagem, 2010; 19(2): 351-7.

11. GENZ N, et al. Doenças sexualmente transmissíveis: conhecimento e comportamento sexual de adolescentes. Texto e Contexto Enfermagem, 2017; v. 26, n. 2, e5100015.

12. GONÇALVES RC, et al. Educação sexual no contexto familiar e escolar: impasses e desafios. HOLOS, 2013; vol. 5, pp. 251-263.

13. LARA LAS, ABDO CHN. Aspectos da atividade sexual precoce. Revista Brasileira de Ginecologia e Obstetrícia, 2015; v. 37, n. 5 , p. $199-202$.

14. LIMA FCA, et al. A experiência e atitudes de adolescentes frente à sexualidade. O mundo da Saúde, 2013; 37(4):385393.

15. MOLA R, et al. Uso de preservativo e consumo de bebida alcoólica em adolescentes e jovens escolares. EINSTEIN, 2016; v. 14, n 2, p. 143-151.

16. MORAES L, et al. Iniciação sexual precoce e fatores associados: uma revisão de literatura. Psicologia, Saúde e Doenças, 2019; v. 20, n. 1, p. 59-73.

17. MORAS SP, VITALLE MSS. Direitos sexuais e reprodutivos na adolescência: interações ONU-Brasil. Ciências e Saúde Coletiva, 2015; 20(8):2523-2531.

18. NERY IS, et al. Abordagem da sexualidade no diálogo entre pais e adolescentes. Acta Paulista de Enfermagem, 2015; v. 28, n. 3, 2015, pp. 287-292.

19. OLSEN JM, et al. Práticas contraceptivas de mulheres jovens: inquérito domiciliar no Município de São Paulo, Brasil. Cadernos de Saúde Pública, 2018; v. 34, n. 2, e00019617.

20. ORCASITA LT, et al. Sexualidad en hombres adolescentes escolarizados: un análisis comparativo entre instituciones públicas y privadas en Colombia. Revista Ciencias de la Salud, 2018; v. 16, n. 3, p. 424-443.

21. Pesquisa nacional de saúde do escolar: 2015 / IBGE, Coordenação de População e Indicadores Sociais. - Rio de Janeiro: IBGE, 2016.

22. PORTELA NLC, ARAÚJO LP. Conhecimento e prática dos métodos contraceptivos por estudantes adolescentes: um estudo comparativo. Revista Univap, 2013; v. 19, n. 33.

23. QUEIRÓS PS, et al. Concepções de pais de adolescentes escolares sobre a sexualidade de seus filhos. Revista Rene, 2016; 17(2):293-300.

24. SOUSA BC, et al. Comportamento sexual e fatores associados em adolescentes da zona rural. Revista de Saúde Pública, 2018; 52:39.

25. VALLI GP, COGO ALP. Blogs escolares sobre sexualidade: estudo exploratório documental. Revista Gaúcha de Enfermagem, 2013; 34(3):31-37.

26. VASCONCELOS ACS, et al. Eu virei homem!: a construção das masculinidades para adolescentes participantes de um projeto de promoção de saúde sexual e reprodutiva. Revista Saúde e Sociedade, 2016; v. 25, n. 1, p. $186-197$. 